\title{
Professional Doctorate Degree Completion
}

National Cancer Institute

\section{Source}

National Cancer Institute. Professional Doctorate Degree Completion. NCI Thesaurus. Code C70558.

Indicates that a person has received a degree allowing them to practice a profession, such as law, medicine, music, or ministry. 